\title{
Valentino Braitenberg: \\ From neuroanatomy to behavior and back
}

\author{
Mario Negrello
}

Received: 22 April 2012 / Accepted: 15 October 2012 / Published online: 13 March 2013

(C) The Author(s) 2013. This article is published with open access at Springerlink.com

\begin{abstract}
This article compiles an expose of Valentino Braitenberg's singular view on neuroanatomy and neuroscience. The review emphasizes his topologically informed work on neuroanatomy and his dialectics of brain-based explanations of motor behavior. Some of his early ideas on topologically informed neuroanatomy are presented, together with some of his more obscure work on the taxonomy of neural fiber bundles and synaptic arborizations. His functionally informed interpretations of neuroanatomy of the cerebellum, cortex, and hippocampus, are introduced. Finally, we will touch on his philosophical views and the inextricable role of function in the explanation of neural behavior.
\end{abstract}

Keywords Neuroscience - Neuroanatomy · History · Braitenberg $\cdot$ Behavioral function

\section{Prolegomena}

Above great achievements, what brings the community to admire a scientist is a set of characteristics that transcend science and knowledge and approach nobility and wisdom. Science is a human endeavor with the commendable goal of understanding reality, in the hope that advancing science will

This article forms part of a special issue of Biological Cybernetics entitled "Structural Aspects of Biological Cybernetics: Valentino Braitenberg, Neuroanatomy, and Brain Function".

M. Negrello ( $\square)$

Computational Neuroscience Laboratory, Okinawa Institute

of Science and Technology, Onna Prefecture, Japan

e-mail: mnegrello@gmail.com

Present Address:

M. Negrello

Department of Neuroscience, Erasmus MC, Rotterdam,

The Netherlands bring us to better understand ourselves, and ourselves in the world. Through the work of great men, we approach that ideal.

In great minds, tenacity is augmented by the ability to channel the product of observations with clarity and flair. In their descriptions the reality beneath the experiment becomes alive, unencumbered by jargon, wants to speak itself. The constraints imposed by experiment rather than nuisances, appear as scaffolds. For them, nature both suggests and takes precedence over theoretical constructs. As a consequence, their prose often reads as if it would have written itself, as if truth yearned for light.

This article was conceived before the passing of Valentino Braitenberg, in the hope that he would have the opportunity to personally review it. Due to that it will likely at times read as an eulogy, but in that case, I believe, excusably. Braitenberg was a model of scientific attitude, both sober and jovial, inspired and humble, strict, and flexible. Simultaneously able to capture the essentials in an effortless glance, while swiftly accepting the burden of proof. With clarity, he indicated the flimsy, the flaky, the incomplete, the obscure, the messy, the missing, meanwhile stressing the necessary, the inescapable, the solid. Sternly, he offered pondered and polished logical foundations of his beliefs.

The article attempts to characterize Valentino's manner of doing neuroanatomy and neuroscience while enlisting some of his most significant contributions to theory, method, and philosophy of the functional interpretation of neuroanatomy.

With the hope of becoming great, we attempt to mirror ourselves against models. In neuroscience we have great men to mirror ourselves against. Cajal, Sherrington, Varela, Bernstein, von Helmholz, Reichardt, and now Braitenberg. Valentino Braitenberg was a living influence on many, and his legacy will be there for many to discover and learn from. 


\section{Introduction}

During his six decades of nonstop scientific activity, Valentino Braitenberg spanned broad contributions to neuroscience opening vast vistas from the standpoint of neuroanatomy. Meticulous, thorough, systematic, and insightful, he furthered staining methods, proposed correction methods for tissue shrinkage, contributed to our understanding of schizophrenia, exposed the computations of myelin bundles, theorized about information and control, advanced our understanding of the hippocampal anatomy, described, quantified, and interpreted the architecture of the cerebral and cerebellar cortex, provided the most comprehensive quantification of the mouse cortex to date, and if that were not enough, invented robot psychology.

This article compiles an expose of Braitenberg's singular view on neuroanatomy and neuroscience. We illustrate his abilities as a scientific diagrammer, as a taxonomist of connectivities, and his functionally informed interpretations of neuroanatomy of the cerebellum, the cortex, and the hippocampus. Excursi on the philosophy behind his observations and opinions will be interspersed within the text. Because of his towering output, this review will, perforce, have exclusions. Notably, only passing mentions of his contributions to insect neuroethology, his deep insights into theories of cortical function, his theory of language, and the lauded book "Vehicles" (Braitenberg 1984). These are left for the reader to discover. The review emphasizes his topologically informed work on neuroanatomy and his dialectics of brain-based explanations of motor behavior.

\section{The primacy of neuroanatomy}

Lest his readership should forget, throughout his publications Braitenberg indefatigably reinstated that neuroarchitecture is the grounding for the understanding of the brain. As he put it, "Neuroanatomy is what is left when all the problems of the neuron are solved" (Braitenberg 1959). Though prima facie few would disagree, there are significant variations in the different approaches on how to proceed with an interpretation of neuroanatomy. Braitenberg's unique take involved making sense of the duals of constancy and variability by localizing invariances and necessities, those aspects of the phenomenon that remained unchanged with respect to particular transformations, and then relating them to behavioral and organismic function.

Braitenberg was perhaps the first neuroanatomist to adopt concepts of topology as a tool to describe and understand neuroanatomy (Braitenberg and Kemali 1970; Braitenberg $1977,1999,1991)$. The vocabulary of topology provided him with conceptual scalpels to effectively describe differences in neuroarchitecture. Through the sibling concepts of symmetry and invariance, his descriptions obtained a degree of clarity unusual for statements about neuroanatomy. With the set of topological transformations Braitenberg classified the cortices. The cortex has rotational invariance, i.e., slices in any vertical plane rotated in any angle are indistinguishable, and translational invariance in the two tangential directions (locally). The hippocampus is a 2D sheet, with translational invariance in one direction (along the Schaffer collateral pathway), and hence, no rotational invariance, i.e., slices in different orientations can be distinguished by whether they are along the pathway or severing it. The cerebellum has no rotational invariance, but has two mirror symmetries, along the parallel fibers, and perpendicular to them. Furthermore, because the pattern between Purkinje cells and parallel fibers is repeated along the direction perpendicular to the parallel fibers, the cerebellar cortex is essentially one-dimensional. Not limited to vertebrates, analysis of symmetries includes other arrangements, such as the periodic patterns of the retina and lamina, which imply a rotational group with exactly six symmetries. Symmetry features are rich with meaning, and in a later section some paragraphs will be devoted to his inspired suggestions. With such descriptions, it becomes possible to identify subtle differences [for instance, that the hippocampus does not look all that different from the cortex (Braitenberg and Schüz 1983)], and to give discerning criteria between brain organs. Also the appearance of particular symmetries, such as columns in the visual cortex (Braitenberg and Braitenberg 1979) or the barrel cortex of rodents, can be explained with relation to behavior (Braitenberg 1977).

Through the lenses of topology, the distinctions between cortical areas are amplified, and the need of explanation for these disparities highlighted.

Some Symmetry Types (from Braitenberg 1999):

Isotropic networks, with full symmetry (locally). Examples are the amigdala or the caudate nucleus, which are invariant to both translations and rotations. Many subcortical structures are locally isotropic.

Rotationally invariant are the networks that have one preferential direction in which neuronal components are stratified and oriented, as the layers of the cerebral cortex, the optic tectum, or the dentate nucleus of the cerebellum.

Flip symmetry, with two clearly defined directions at right angles to each other-along the third axis the structure repeats homogeneously. E.g., hippocampus, where perpendicular direction dentate gyrus-subiculum, also well defined by the course of hippocampal mossy fibers and schaffer collaterals. 
Lattice, with mirror symmetries and translational invariance as in the cerebellum. Translatory symmetry in one direction, as in the spinal cord.

Periodic, with discrete translatory symmetry, such as in the periodic ganglia of some insects [e.g., the Lamina of the fly, the central complex of sophisticated insects (Strausfeld et al. 1998; Strausfeld 2009)].

\subsection{Topological unfoldings}

Having the brain in the mind's eye can be a daunting task. Even from experts we often get the impression that the brain is a twine knot impossible to untangle. For instance, the basal ganglia or the thalamus seem to be connected to everywhere, and that is often as much anatomy as many neuroscientists will allow. But generalized characterizations of connectivity fail to convey that the namespace anatomists use, relies on, and maps onto, very particular arrangements between brain organs. This is where Braitenberg's understanding of topology has provided more than one of us with mental pictures which allow the mind to grasp many brain areas simultaneously, in relation to each other, as if one had personally performed the dissections. These pictures are ripe with developmental, organismic and evolutionary hypotheses about the relationships between neuroanatomy and behavior.

Braitenberg had pictures of the relationships between brain areas in his mind-pictures that he was able to share through inspired diagrams. His diagrams of unfolding brains and brain parts were an essential didactic instrument in smoothly walking the reader through arguments about information flows in the brain.

In what has become a reference work about the neuroanatomy of the cerebellum (Braitenberg and Atwood 1958), he presented it in an intuition defying picture (Fig. 3), eliminating what is perhaps the most conspicuous macroscopic feature of the cerebellum, its multiple invaginations, the folia. Not satisfied, he inflated the cerebellum into a balloon, deliberately ignoring that the unfolded cerebellum is much longer in the rostro-caudal direction as in the medio-lateral (Fig. 1; see also Sultan and Braitenberg (1993) for fascinating comparative neuroanatomy). This topological transformation produces a revealing picture which emphasized continuities and discontinuities in the cerebellar cortex to great didactical effect. It becomes clear that the cerebellum, in contrast to the cortex, is continuous in the midline, that the vermal cortex is mostly disjoint with the hemispheres, and that the flocculus dangles somewhat isolated from the cerebellar flanks. Subsequently, focusing on the study of the foliation, an inspired diagram (Fig. 2) about density of folia (cerebellar convolutions) reveals subtle and yet regular asymmetries, further explored in relation to cell counts,

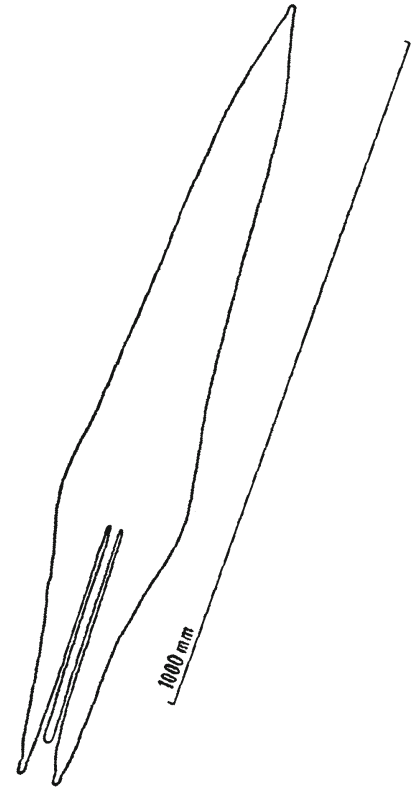

Fig. 1 The thin and long shape of the unfolded human cerebellum (from Braitenberg and Atwood 1958)

fiber bundles, and layer thicknesses. Aesthetic depictions like these lend the reader Braitenberg's eyes, and his intext descriptions complement the pictures with suggestive insights on their hypothetical significance.

No less stunning is his depiction of the unfolding cortical hemisphere of the mouse (Fig. 4b, from Braitenberg and Schüz 1983), where the cortex and hippocampus exhibit their natural border, concealed from sight in standard brain sections, deconvolving the obstacles to the student of neuroanatomy, providing a tool for thought (it is endearing that Braitenberg politely apologizes to the perchance callous neuroanatomist, for whom this might have looked trivial, for this didactic excursus). In the picture, the transition between the occipital cortex through the entorhinal cortex to the subicculum to the hippocampus, and on to the fascia dentata, becomes obvious (Fig. 4c). The hippocampus appears as the opposite pole from the olfactory cortex, indicating its evolutionary origins (Fig. 4a). Complementing this picture, a second diagram displays clearly the fascia dentata as a sheet (fascia) enveloping and providing a terminal cap to the hippocampus. Through this topological picture, interpretation of the involved convolutions of the hippocampus as they appear in standard sections becomes natural. The sparse and tridimensional layers of the cortex are progressively squeezed into the subiculum, becoming almost planar in the hippocampus. The fascia dentata enwraps the thin edge of the hippocampus. Such visual understanding invites speculation on the activity fluxes to and fro this flummoxing piece of neural machinery. It also invites speculations relevant to embryogenesis, phylogeny or comparative neuroethology (more about this in Sect. 4.4). 


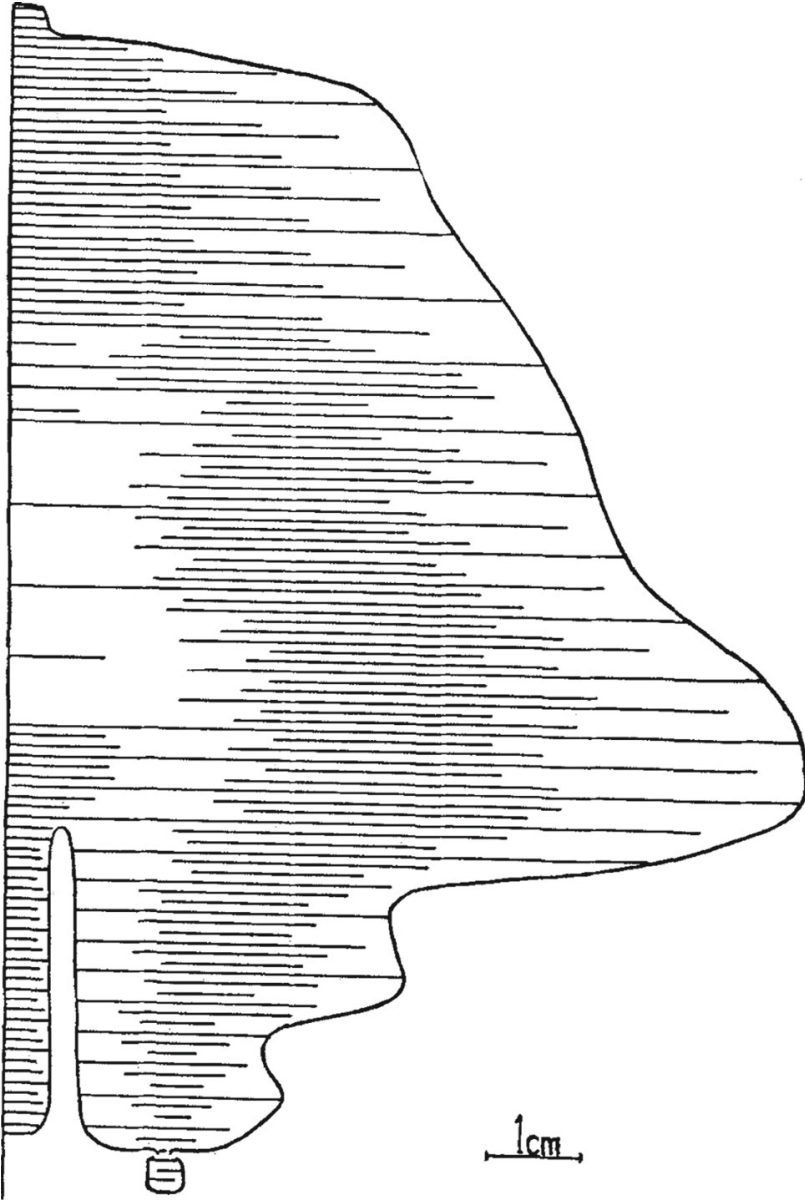

Fig. 2 (original text) Plane representation of the right half of the cerebellar cortical sheet. Horizontal parallel lines have the actual measured length of the folia; three lines represent ten folia. Actual vertical distances in this representation can be obtained by multiplying number of horizontal lines by $10 \mathrm{~mm}$ (from Braitenberg and Atwood 1958)

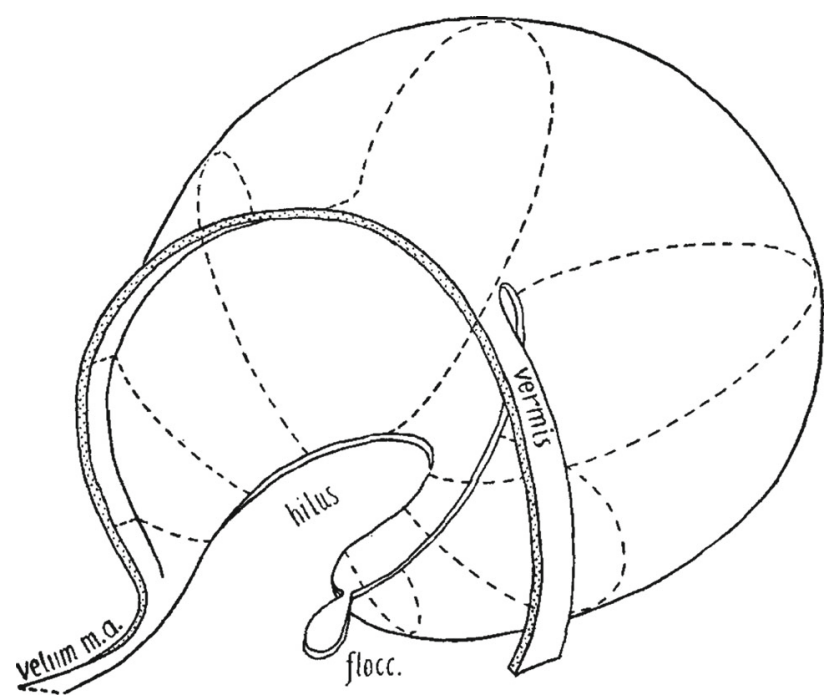

Fig. 3 The inflated cerebellum (from Braitenberg and Atwood 1958)

\subsection{Meaning in numbers}

Alongside with geometry and topology, also numbers are deconvolved to provide qualitative insight in Braitenberg's articles.

Methodically, Braitenberg deployed traditional methods in neuroanatomy to count neurons, synapses, glia, and fibers. Facing inadequacies of these methods he furthered them, introducing alchemic variations of the Golgi, Nissl, and fiber tract stains, revealing neuroarchitecture at many scales (Braitenberg 1978; Braitenberg et al. 1967; see also Chapter 3, of Braitenberg and Schüz 1998). From these preparations, Braitenberg (often assisted by his long-term collaborator Almut Schüz) painstakingly compiled counts of neurons and glia, estimates for axonal and dendritic lengths and branchings, volume occupancy of different cortical elements, buttonic and synaptic densities. In their 1998 book "Cortex: statistics and geometry of cortical connectivity", Braitenberg and Schüz (1998) present the results of decades of research in a comprehensive resource of the mouse's brain, which provides an extensive compendium of numbers, invaluable resource for modelers and neuroscience community, an admirable feature of which is the many ways in which estimations are derived in order to reciprocally verify estimates from diverse routes (Fig. 5).

The book exemplifies Braitenberg's take on the meaning of numbers, offering simple yet compelling explanations for constancy, variability, and scaling.

Why should the square root of brain weight be proportional to the cubic root of body weight? A clue is given by the scaling rule of sensory sheets (2D), with brain volume (3D). ${ }^{1}$ Why should neuronal density be inversely proportional to the cubic root of brain volume, or why is the cell density smaller in elephants than in mice? Look at the length of axons connecting areas for the answer. Are there interesting exceptions for scaling rules? Where do they occur? And why? Measure the different brains and regard the evolutionary ecology of their owners to look at inter-areal differences.

In my personal experience, it is not uncommon to meet neuroscientists (especially in the synthetic and computational side of things) that know no numbers about the brain, but two. The number of neurons and synapses of the human brain. Rather than explanatory devices, these two numbers are often presented to impress the audience (though the impact has diminished over time). Cell counts, however, become most useful in the comparative context: how many motor neurons are there in relation to a particular muscular innervation? How do cell counts scale with species size, and what is the relationship to axons and dendrites, and how this relates to

\footnotetext{
${ }^{1}$ Interestingly though, many of his cogent results have remained in relative obscurity, given the fact that some have attempted to reinvent the wheel [refs], albeit never with similar clarity.
} 
Fig. 4 The unfolded hippocampus (from Braitenberg and Schüz 1983) a

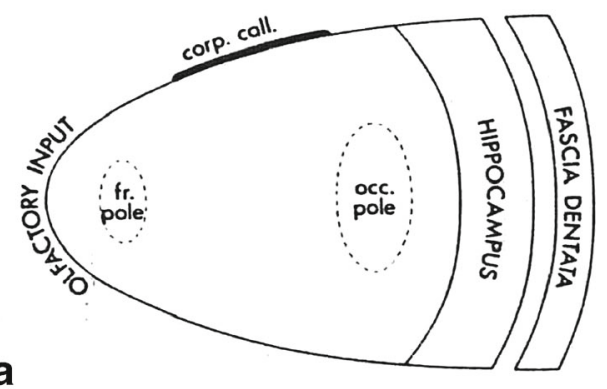

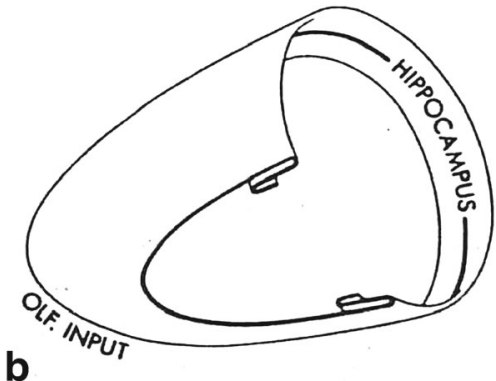

b

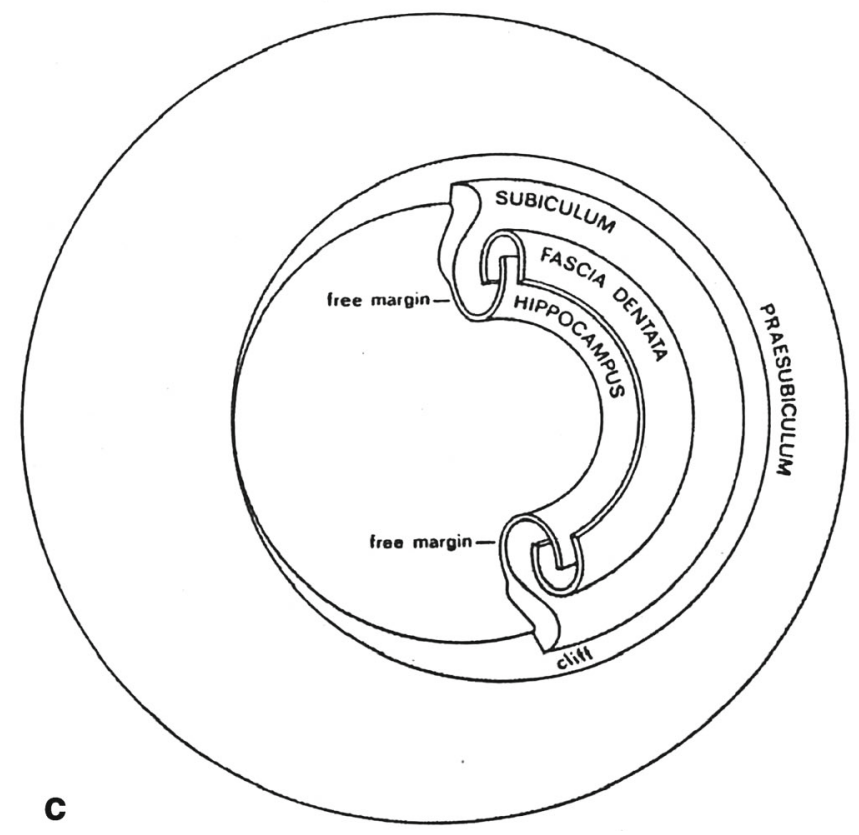

Conclusions

Deduced quantities

Measurements

No. of sensory input fibres $<10^{\circ}$

Vol. (iso- and allocortex) $2 \times 87 \mathrm{~mm}^{3}$

Density of neurons $9 \times 10^{4} / \mathrm{mm}^{3} \longrightarrow$ Total neurons $1.6 \times 10^{7}$

Density of

Density of synapses $7 \times 10^{8} / \mathrm{mm}^{3}=$

Average distance of synap. on axons $5 \mu \mathrm{m} \longrightarrow$ Syn./length of axon $200 / \mathrm{mm} \longrightarrow$ Density of axons $4 \mathrm{~km} / \mathrm{mm}^{3}$

Length of the axonal tree $10-40 \mathrm{~mm}$ Rel. density of axons (synapses)

Range of axons: pyramidat small stellate cell $0.2 \mathrm{~mm}$

Density of dendrites $0.4 \mathrm{~km} / \mathrm{mm}^{3}$

Length of dendritic tree $4 \mathrm{~mm}$

Range of dendrites $0.2 \mathrm{~mm}$

Spines/unit length of dendrites $1-2 / \mu \mathrm{m}$

(synapses on spineless dendrites $3 / \mu \mathrm{m}$ )

Percent py-cells $85 \%$

(1)

Percent Type-1-synapses $89 \%$

$\begin{array}{ll}\text { pyramidal } & 10^{-5} \\ \text { stellate } & 10^{-3}\end{array}$ afferent $10^{-3}$

Rel. density of dendrites $10^{-3}$ (Py., basal dendrites)

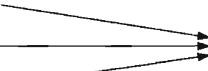

um

Probabily of synapses bet

2 py-cells $0.2-0.3 \mathrm{~mm}$ apart:

0 syn. $p=0.9$

1 syn. $p=0.09$
2 syn. $p=0.004$

Percent synapses on spines $75 \%$

Fig. 5 Summary of measurements, deduced quantities, and derived conclusions (from Braitenberg and Schüz 1998) 


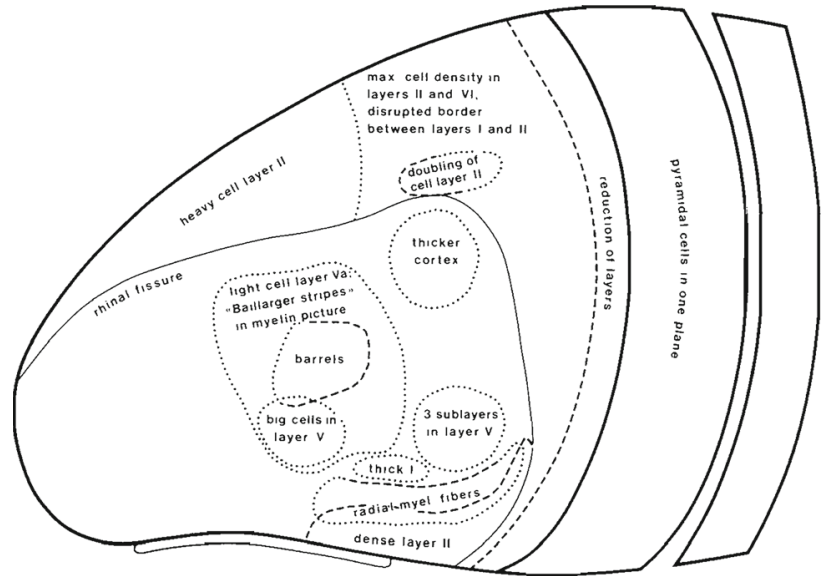

Fig. 6 Map of cortical layers of the mouse cortex. Note the assumed continuity between outlined areas (from Braitenberg and Schüz 1998)

cross-species scalability? How many neurons are found in the olfactory cortex, presumably the precursor of the thinking brain, of different species? Through cunning quantifications, many such questions admit productive speculation, and to the extent that knowing such numbers is not sufficient to understand the brain, without them it becomes an exercise in arbitrariness.

He shows us that from first principles it is also possible to derive general statements about the organization of the cortex, and the invariance of certain cell ratios, as for instance, the relationship between the two-dimensional surface of the sensory sheets and the three-dimensional volume of the brain. Braitenberg (2001) shows that modular connectivity is sufficient to explain a number of scaling relationships between body mass and brain volume, an explanation presaged in his early paper on the morphology of nerve nets (Braitenberg 1959). Examining the types of connectivity rules that can exist (all-to-all, local, modular, etc.), and despite idealizations, the scaling exponent for body mass and brain volume is revealed from a savory set of simple equations, whose derivation can be understood by anyone with high-school math.

\subsection{Cytoarchitectonics}

His cytoarchitetonic maps of the mouse cortex are not the cartographical maps of Broca or Wernicke. Rather, his maps focus on relevant differences and gradations between them. Like distinguishing Italians from Austrians, by starting with the stereotypes. But as in geopolitics of Italy and Austria, the Alto Adige and the Süd Tirol can be one and the same, though different, dependent on contextual stance. Much can be learned from archetypes, and just as much from the gradual miscegenation from one into the other (Fig. 6).

Numbers and cytoarchitectonic maps often have functional concomitants, closely dependent on the ecological setting and evolutionary history of beings. Through Braitenberg's lenses, even the deceivingly pedestrian cell counts teem with meaning. But it is in the functional interpretation of structures that we find some of his most compelling observations.

\section{Functions of structure}

\subsection{Structural deficit, functional pathology}

It is befitting that one of Braitenberg's earliest interests was the neuroanatomy of schizophrenia (Braitenberg 1954), arguably the most peculiar of mental ailments due to its vast and bewildering symptomatology, in which the subtlest changes of wiring, eluding even modern day tractography, lead to the most surreptitious behaviors, implying devious links between brain structure and cognitive behavior. Ever since his first article where he, as a young medical doctor, had the opportunity to examine Vogt and Vogt's collection of schizophrenic brains, noticing the exquisite subtlety of variations of the insidious condition, had a keen sense of the tight relationship between structure and function. That article bespeaks, despite the immense gaps of knowledge at the time, his penetrating insights on the minute disparities in cell counts and myel$\mathrm{o}$-architectonics, the analysis of which produced ideas made even more timely by modern advances in measurement, such as diffusion tensor imaging (DTI). Not long afterwards he devoted himself to the study of myelo-architectonics and the functional implications of fiber bundle patterns, summarized next.

\subsection{Connection schemes and myelo-architectonics}

For scientists of this materialistic age, it is a truism that function derives from structure (and the dynamics defined therewith). The struggle is in inferring the one from the other. While most would start (and many would remain) in the cortical grey matter, Braitenberg emphasized that much foundational understanding could be obtained by the study of the, often unsung, white matter bundles. The white and fatty insulated bundles are formed by axons of neurons as they traverse between multiple brain areas, in the path to their targets. Myelinated bundles are more than the source of whale oil. This intricate set of fibers, whose paths are anything but arbitrary, can be examined even from macroscopic features to expose the putative computations performed.

Through visual examination we can determine, for instance, whether bundles converge or diverge simply by observing the relative thicknesses of the fiber bundles arriving and leaving areas. Functionally, a classification machine able to categorize any spatial pattern would show great divergence (in recent literature represented sparsity and 
decorrelations (Franzius et al. 2007; Wyss et al. 2003)), while a device transforming spatio in temporal patterns would show great convergence [a connectivity pattern that is at the core of echo-state networks and liquid-state machines (Yamazaki and Tanaka 2007)]. Should we be amazed that mechanistic hypotheses can be deduced from macroscopic features of the brain?

Myelinated fiber bundles originate and project to areas in a way that, to the chagrin of box diagrams, is exquisitely heterogeneous. Many of these patterns are distinguishable with the naked eye, where one is able to identify, with the help of myelin staining, and more recently with DTI tractography, an assortment of patterns: taperings, commissures, decussations, chiasms, branchings (Fig. 7). Such visible features can be interpreted in terms of the topography of fiber connections, and Braitenberg, in a paper that could have been written by a bright undergraduate, catalogued such patterns showing how the observable features of fiber bundles could uncover connectivity patterns (Braitenberg 1962).

Further, Braitenberg pointed out that the very mappings resolved by simple fiber patterns could perform computations. As it became customary for him, he systematically enlisted and exemplified patterns, interpreting them in terms of the computations they could imply. In his manner, and in interest of completeness, he also added patterns to the list which do not exist in nature.

In addition to fiber tracts, also the shape of synaptic field terminations, what is now commonly referred to as arborizations, can be characterized and taxonomized in classifications that expose function, or at least nourish speculation. Such lists help the mind to encircle the spectrum of possibilities, and from contrasts, to calculate implications.

Taxonomy of synaptic fields

- Fields of excitation nearly coinciding with the pickup field (granula of the cerebral cortex).

- Far removed from the pickup field as in the giant pyramidal cells (Betz cells) of the cerebral cortex.

- Very dense branching within a narrow region (incoming sensory fibers in cortex).

- Diffuse branching over large regions (reticulo-cortical neurons).

- Long straight unbranched and uninsulated (the example is left as an exercise for the reader. Hint: timing!).

- Chains of separate fields of inhibition (basket cells of the cerebellar cortex).

- Common shapes are spherical, flat box, or an elongated pear (most frequent in the cerebral cortex).

In a time where so much knowledge about the brain is given in conceptually immaterial entities, it is relieving to see that some entities do indeed exist with palpable counterparts. What is more, that even macroscopic patterns such as those of fiber bundles can inform us about the transformations that neural activity may undergo.

Of the bundle fiber patterns, perhaps the one that has most occupied his theoretical endeavors were decussations-fiber crossings that connect non-homologous parts of the brain (contrast with commissures). His keenness to provide computational interpretations of neuroanatomy is symbolized by his study of taxis, decussation and kynesis (Braitenberg 1965), an early precursor to the widely acknowledged vehicles (Braitenberg 1984). In it, we find the seed for his take on the links between structure and function. His suggestions helped shape a wealth of disciplines, from the interpretation of neuroanatomy, artificial life and embodied robotics, to philosophy of mind. Because this is perhaps his best known work, we shall not be detained here. Suffice it to say that Braitenberg's work on the analysis of the white matter should remind neuroscientists that not all is grey.

\subsection{Brain organs}

Because functional inferences suggested themselves to Braitenberg, the observation of architectonic peculiarities was frequently adjoined by a functional hypothesis or cunning speculation. The following sections feature some selected examples, on the hippocampus and cerebellum, emphasis, his and mine, on the latter.

\subsection{Hippocampus}

Though never having been a primary subject of research, Braitenberg's comments on the Hippocampus strike the naive reader as foundational. This joyful and almost poetic abstract in one of the few papers published on the topic bespeak a fascination with the topic:

A beautiful appearance under the microscope, a striking biochemistry, a miraculous electrophysiological approach as well as a peculiar psychological relevance make the hippocampus the favorite subject for diverse groups of enthusiasts. (Braitenberg and Schüz 1983)

As his signature style, Braitenberg compiles a list of the hippocampus's unique features, from cytoarchitectonics, through the unique features of its circuitry, to comparative neuroanatomy and evolutionary history.

Architectonical comments:

- Only the lower part of the cortex (layer VI) is continuous with the hippocampus.

- Entorhino-hippocampal pathway is directional, one way, not matched by a similar on the other direction. His interpretation: igniting of cell assemblies.

- The flow of information (through the perforant fibers, mossy fibers and Schaffer collaterals) from the upper 
Fig. 7 Some branching patterns, their potential functions and some examples (adapted from Braitenberg 1962)
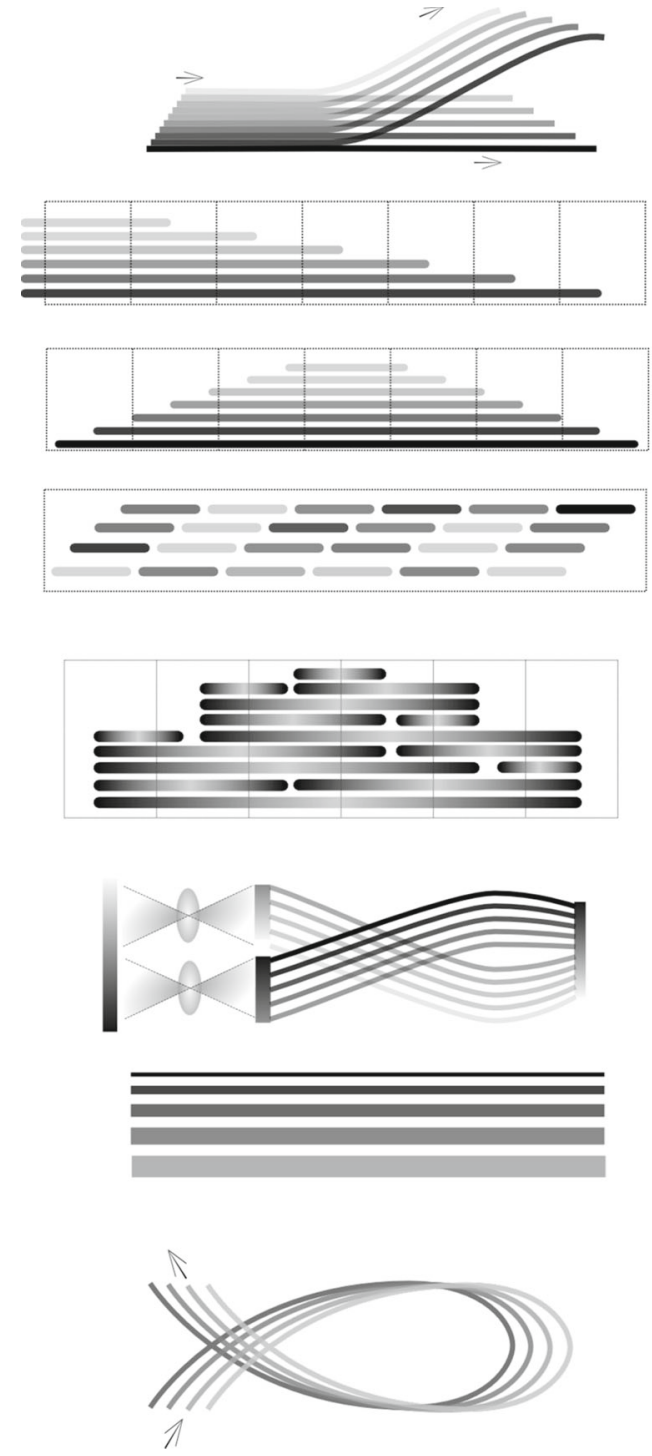

Branching bundles: Translation, multiplication and delay of patterns of excitation. In other cases the bifurcation is the endpoint of axons, which may be able to gate transmission.

Tapering bundles: Frequent pattern. Correspondence between target areas and subdivisions of an organ. Examples: fimbria of the hippocampus, optic fibers in lower vertebrate tectum, bundles in the lateral thalamus.

Bilaterally tapering bundles: Frequent pattern in vertebrate brains. Indicate a commissure. Mirror Symmetry. Comes in two sorts:

a.A system of fibers, systematically connecting areas within the same organ. Ex: Horizontal fibers in layer 4 of the striate area (now V1- these have later been associated with surround inhibition).

\section{b. A system of fibers connecting each subdivision with every other subdivision. A classification machine (one that classifies all possible binary patterns would have a $\mathrm{n}-\mathrm{n} 2$ relationship). Possible to find in the inner stria of Baillarger or in the primary acoustic area on Herschl's convolution.}

Crossing fiber bundles (chiasms) Optic Chiasm (many others) If two halves of a surface are connected with the corresponding halves of another, the splitting of the pattern annuls the disruption of continuity of a pattern of excitation, indicating reconstitution of a continuous pattern.

Bundles with Fibers of different thicknesses. Not observed during at his time, but he pointed out that parallel Fibers of the cerebellum have different conduction velocities (and later were found indeed to have different thicknesses). Different velocity of conduction produces temporal scattering.

Loops can function as delay organs. Not existing in nature? layers of the entorhinal cortex toward its extended sixth layer (the subiculum-hippocampus) does not follow the direction one would expect, but skips to the extreme ranges of the protruding tongue and then proceeds backward in the direction of the entorhinal cortex.

- System of unidirectional connections in the entorhinohipocampal loop (not seen anywhere else in the cortex). Not numerically prevalent but strong influence from dentate to CA3 connections because of their placement on a crucial part of the dendritic tree.

\section{Evolutionary Comments:}

- A criterion for the identification of hippocampi in nonmammalian brains: where there is no path topologically identical to the perforant path, there is no hippocampus.
- The hippocampus arose from its output, the olfactory cortex.

- There is no markedly two-dimensional hippocampus in fishes which live in 3D space. Incidentally, among the mammals, the dolphin has a surprisingly small hippocampus.

Linking with the results of O'Keefe and Reece (O'Keefe and Dostrovsky 1971; Jeffery and Burgess 2006) he writes: "A failure to detect a clear isomorphism between the hippocampus and the external space to which they refer works against his idea, but such an isomorphism may still be found." And indeed it seems to have, as recent experiments evince topologically connected place fields and grid cells, validating his foresight. 


\subsection{Cerebellum and motion}

If the hippocampus attracted Braitenberg for its structural uniqueness, the cerebellum and Braitenberg were predestined to each other. In no other brain structure does one find such remarkable symmetries and invariances. Moreover, the organ of motion has a particular functional relevance for the organism as, quoth Sherrington, "The only way to do things is by moving ourselves, the world, or ourselves in relation to it". Braitenberg's life-long fascination with the cerebellum was never dissociated from the output, motor behavior. From his early suggestions on to the sophisticated views he sported more recently, the boundary conditions for his cerebellar theories were always given by the reciprocity between neuroanatomy and the physics of movement.

It would be hopeless in this review to encompass the multitudinal character of Braitenberg's endeavors in the physics of movement and theories of motor control, but it should be possible to gist his methods, and enlist some of the most significant contributions. The original treaties far surpass anything I can offer, and should I commit any injustice, as an apology I point to the sources.

In many discussions of cerebellar function, Braitenberg started by comparing the cerebellum and the cerebral cortices (Braitenberg 2002; Bower 2002; Braitenberg et al. 1997). Contrasting the cerebellum and the brain may seem ludicrous at first, but the exercise reveals curious similarities and informative disparities.

Cerebrum and cerebellum:

- Convergence and Divergence the cerebellum displays remarkable convergence and divergence (output of the cerebellum is on the order of $10^{7}$, same order for the input). In the telencephalon, the sensory input is on the order of $10^{6}$ (from which the visual is the lion's share), with the same order for the motor output (pyramidal tract). The divergence between input and interneurons in both systems is given by a factor of $10^{4}$.

- Synapses in the cerebral cortex: $10^{14}, 10^{4}$ on the $10^{10}$ pyramidal cells and fewer on the stellate cells. Synapses in cerebellum: a few times $10^{2}$ on the axons of the $10^{11}$ granule cells, a few times $10^{5}$ on dendrites of $10^{7}$ purkinje neurons, $10^{13}$ on the other inhibitory cells. Proportion of synapses on spines: $85 \%$ in cerebellum and $75 \%$ in cerebral (some take this as a measure of plasticity).

- Inhibitory Neurons reveal a surprise: percentage of inhibitory neurons, in the cerebellum $1 \%$, in telencephalon $15 \%$.

- Structural Traits and Symmetries Both are flat, and have comparable area in humans. The cerebellum is uniform throughout, with the exception of the floccular cortex. In any small fraction of the cerebellum two directions are always defined (it is anisotropic, with two flip symmetries), for every fiber running in the $x$ direction (along the parallel fibers ${ }^{2}$ ) there is one in the $-x$. Same is valid for $-y$. In the cortex, locally, only one direction is anisotropic (across the layers). In the cerebellum, there are no cortico-cortico connections in the white substance underlying the cerebellar cortex. And there are no intrinsic excitatory fibers. Unlike the cortical input, the two kinds of input fibers into the cerebellum are radically distinct. Climbing fibers select individual neurons, mossy fibers reach extended and overlapping regions. Both are compartmentalized, but with distinct symmetries.

From these and other facts, he offers a wealth of deductions about cerebellar function: basic transformation is the same in different contexts (motor, vestibular). Input spreads in two directions, and interactions are local. The principles must be embodied in a mesh no larger than a few millimeters each side. Interactions in opposite directions have the same effects. Climbing fiber reaches the output directly in a point-to-point fashion. Mossy fibers excite extended, discontinuous patterns of separate points. Activation of the granule cells in one point can only produce excitation in a small percentage of cells. Not a beam (as in Eccles), but tides (i.e., the beam theory could not work, "if for no other reason but that the term suggests something strong and inflexible". ${ }^{3}$ ) Braitenberg suggested 'groove' instead of beam to represent the tidal summation (Fig. 8). "Parallel fibers acquire their individuality by virtue of the system of inhibitory fibers arranged at right angles", supporting spatio-temporal summation in the parallel fiber system.

Many of these deductions had been available and known to cerebellar scientists at the time. Many schemes were proposed to encompass the known anatomy and physiology of the cerebellum, most famously the "Marr-Albus-Ito" theory of cerebellar learning. But Braitenberg's own theory has the desirable features of respecting anatomy, as it takes up the challenge of explaining motor control under the assumption that it has to explain movement as the organism does it, under the constraints and capabilities with which it is endowed.

\footnotetext{
${ }^{2}$ Despite being a neuroanatomist, Braitenberg suggested that the system of coordinates to speak about the cerebellum, instead of the artificial 3 plane section commonly applied to the cortex, should be given in the system of coordinates most natural to the orientation of fibers ( $x$ along the parallel fibers, $y$ perpendicular to them, and $z$, depth). Recent advances in developmental neuroanatomy, as in the work of the anatomist Luis Puelles, also suggest coordinate systems more related to morphogenesis, as the geometry can best be understood in the context of self-organization of organismic function.

3 Interestingly, many 'disproving' arguments against the 'grooves' are directed towards the old 'beams'.
} 
Fig. 8 Grooves of excitation (from Braitenberg 1987)
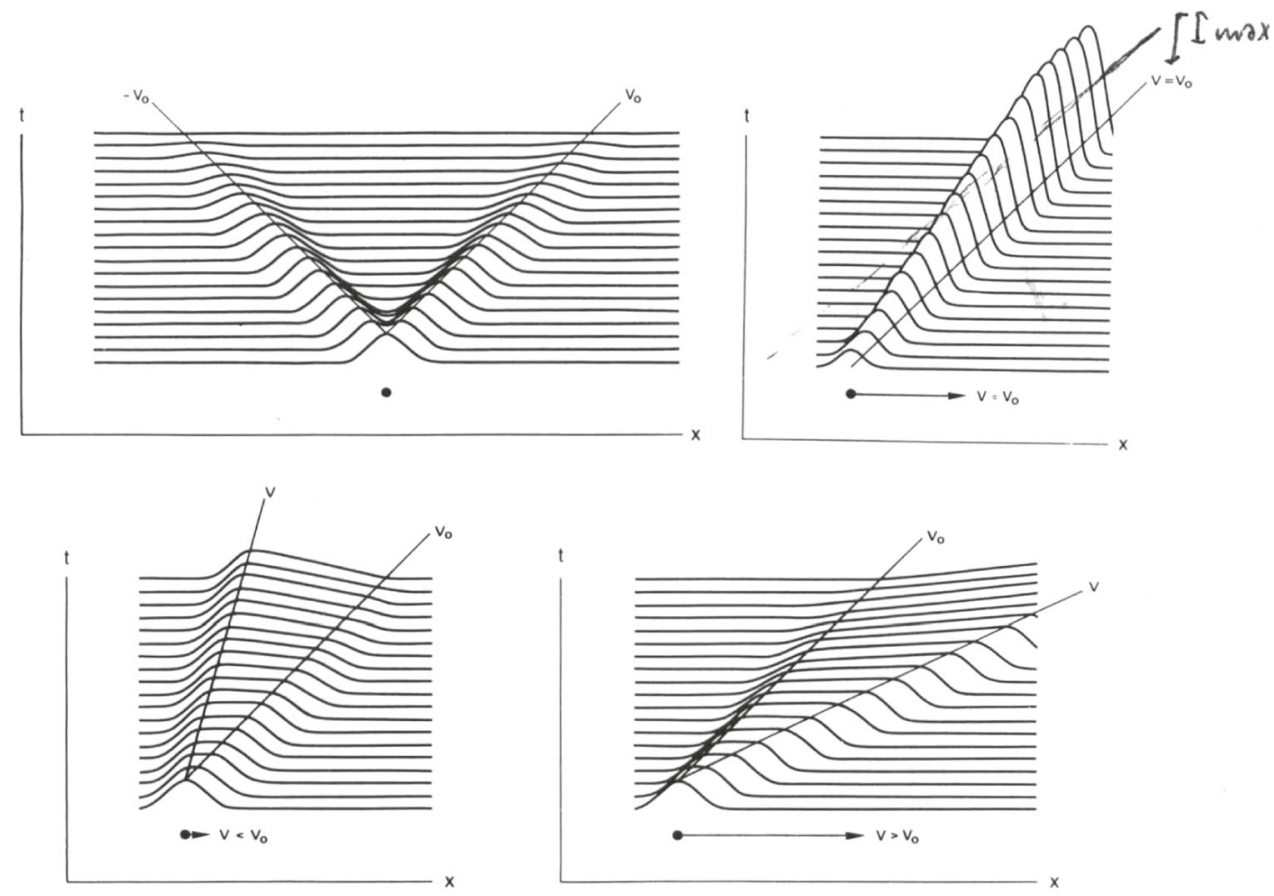

Fig. 2. Waves of excitation propagating in the molecular layer of the cerebellum (hypothetical). The direction of the parallel fibres is represented in the horizontal $(x)$, time runs upward $(t)$. Upper left: a pulse of excitation (black dot) sets up two waves running in opposite directions and extinguishing when the end of the parallel fibres is reached. Upper right: A stimulus moving at the intrinsic velocity $v_{0}$ sets up a "tidal wave" of maximal amplitude, whereas different velocities (lower left and right) produce lesser waves.

\subsection{Inventing the cerebellum}

Cerebellum concerned with movement was established early in the history of neuroscience with Rolando and Flourens in the first half of the nineteenth century, as lesions were reliably associated with ataxias of diverse symptomatology of disturbances of control, sequencing, and coordination of movement. Braitenberg's ideas on the topic were scattered in his scientific output throughout decades, and articles devoted to it often warned the reader in their titles of the hypothetical character with the words "The cerebellum and the physics of movement, some speculations", and "Intricacies of motor control. An essay". Despite his emphasis on the word "speculation', this should be taken as a sign of humility, for many of his insights are logical consequences of premises well established in fact.

Braitenberg's article "Intricacies of movement control" departs from a taxonomy of animal movement, that surveys the phenomenology of motion generation, from the undulatory movement of worms to the subtle and sensitive touch. Thereafter, he indulges in inventing a machine for motion control, which bears a remarkable resemblance with the cerebellum.
I would like to quote the introduction to that paper in its entirety (the whole paper is quotation worthy material):

The synthetic approach of robotics and the analytic approach of motor physiology have not yet reached much common ground. To be sure, some ideas first formulated in engineering have entered the vocabulary of the physiologists (e.g., position feedback, bang-bang control, stability, frames of reference) thereby making the writings of each camp more palatable to the other. However, the engineers still regard the biological system as just one possible solution among many, almost certainly not the most efficient one. Conversely to the physiologist, the robot looks much too clumsy to be ever taken seriously as an approximation of the real thing.

I believe that we could come closer to each other if each would actively invade the other's territory, the physiologist inventing machines unhampered by restraints of technical feasibility, and the engineer explaining the brain in ignorance of the (indeed scanty) physiological evidence. I will do a bit of both, putting myself in the role of both kinds of trespassers, combining so to say 
two kinds of ignorance in the hope of achieving some fruitful synthesis.

"Inventing the cerebellum" (section 3 of Braitenberg 1993 ) is a study in reverse engineering, considering the kind of tasks that an organ of movement has to solve. It departs from a taxonomy of the kinds of movements that organisms exhibit. A theory of the cerebellum has to account for them, as it interprets its anatomy in accordance. His list distinguishes types of movement that span the whole phenomenology. Movement, broadly, is a change of the position of parts of the animal relative to each other. It is to be distinguished from locomotion, when the position of the animal changes relative to external coordinates. Movements are further categorized in deformations of the body, and movement against an external force. Deformations contain the categories of symmetrical (as extending limbs simultaneously), or asymmetrical (with respect to the center of gravity), such as moving a leg or an arm at a time. Rotation is an example of an asymmetrical movement, of which there are multiple kinds: rotation of a limb with respect to the body, or flexion and extension, such that the angular momentum cancels each other (anti-symmetrical). Another kind of asymmetrical deformations is given by swinging an appendage on its hinge, which produces a rotation of the bulk of the body (fly wheel motion of the cat). In oscillation, opposing forms are mostly internal. Turning to deformations against external forces, we have posture in which the whole body stands against gravity, and pendular in which appendages have to oppose it. Pushing and Pulling, happen when two bodies are involved, or when locomoting (pushing against friction). In addition, Jumping and Throwing, when the contact between agent and object is interrupted, and Touching and Holding, where the forces are given by the elastic deformation of tissue.

These problems are general problems of movement, of which most are associated, in vertebrates, with cerebellar function. These are the explananda. On the basis of the physical requirements for movement, and interpreting anatomical features, Braitenberg proceeds to 'invent a cerebellum'. "My plea is for the simples possible principles of mechanics to be invoked in the explanation of movement since the motor system, in order to be efficient and economical, cannot but conform to such principles". His desiderata for a contraption that would potentially solve the problems of movement are enlisted in the end of the article. It seems appropriate to put them in bullets. If he were inventing a control system for movement, he would have it in this fashion:

Two main tasks are to be distinguished, and assign one mechanism for each. One mechanism for Action, deliberate movements of the body that change the state of the world to conform to a desired picture. The other is stability mechanism, whose role is to maintain the body stability by compensating for the physics of the body. The stability machine would perform two kinds of translations of internal states, efferent and afferent. Efferent states translate the states of the internal elements into signals to the muscles which activate them exactly to the degree that corresponds to theirs state of contraction in the internal representation. Afferent states will signal deviations from the internal representations and can then be used to assess external forces that are in the way of the motor realization. In this view, the position of the body is an automatic consequence of 'thinking' that position. (as in the proposition of equilibrium point control by Feldman (1986) and Bizze et al. (1982)). The computer of stability could in principle be informed about every transition planned by the cognitive machine. Incorporating knowledge of the laws of mechanics and of the quantities involved (mass shape elasticity, viscosity), it could in principle compute all the deviations from the planned action and produce the corrections in real time. The stability machine would have implicit representations of the variables of movement, for explicit representations would be verbose and cumbersome. "Since many different situations have to dealt with for which there is no common solution, I would compartmentalize my machine. The stability machine does has sequences as input and must associate to each particular output sequence. My machine would work in the 'sequence in sequence out' mode." Signals would come in different combinations and at different locations (signifying different phases), and therewith diverse combinations of motor ensembles. Outputs would be combined with appropriate delays to activate the motor elements. A guided learning process to transform angular and overshooting movement into elegant and precise movements, by selecting well-timed action. The machine should save the organism's energy, by avoiding unnecessary action, while being responsive to urgent action.

Humorously, Braitenberg proceeds to point out that the machine he has been inventing has many features in common with the cerebellum, by assigning roles to the notable cerebellar components: its elongated compartments of parallel fibers, the individuality of compartments conferred by the baskets, and stellate interneurons arranged at right angles. That a large part of the cerebellar input comes from the cortex 'the cognitive machine' via the pontine nuclei (and now we know, also through the basal ganglia). Purkinje cells receive connections from the parallel fibers from a multitude of synapses, in accordance with the learning requirement. And finally, the output is inhibitory, in agreement with an energy saving principle.

To the extent that many of these insights have now unconsciously made their way into the cerebellar field, the rich and coherent picture Braitenberg painted through his career is foundational, but strangely unacknowledged. Although these collected thoughts appear in the 1986 article, many of these views were present even in his seminal 1958 article, and many subsequent ones in which he more fully 
developed quantitative and relational theories of the principles involved.

\subsubsection{Cerebellar necessities}

It is perhaps not a mystery that his life-long interest dwelt in the interpretation of the cerebellum. In ' 83 , he wrote " 25 years ago I thought that the cerebellum was the part of the brain most likely to be understood by direct translation of the anatomy into functional terms. Today I am still confident, even if the schemes proposed in the meantime have proved to cover at best only very partial aspects of cerebellar function and sometimes quite divorced from any realistic consideration of histology" (Braitenberg 1983). Devotedly, he honed his explanations and interpretations of the cerebellum, integrating new thoughts and new results as they came into a framework.

And despite the often heard trite dismissal of the cerebellum being merely the organ of motion (as if this would mean it's uninteresting!), we still lack a theory that satisfies both histology and motor behavior. The case may still prove to be that Braitenberg's explanations will be a component of the successful ones to come. That the cerebellum is the organ of sequencing, that parallel fibers will still turn out to act more or less as delay lines, and that compartments will stand for spatial temporal transformations of specific muscle groups are still tenable hypotheses, notwithstanding the vociferous criticism often received, as Braitenberg's flights of fancy were firmly grounded on knowledge of fluid mechanics.

In his words "I am reluctant to give up an idea on neural computation which has the rare merit of providing detailed explanations of many morphological peculiarities in a piece of grey substance". Rightfully so, as his consistency afforded him a sophisticated system of understanding, perhaps one of the most distinguishing features of his work. Whether his hypotheses are verified remains to be shown, but whatever the case, the shape of an explanation will have to abide to his observations bearing on necessities. One of the core necessities is that the outside, the world, and the inside, the brain, must meet.

\section{Conclusion: inside out and outside in meet}

Let us indulge, and imagine for a second that we at present already possess all the knowledge about the brain that would allow us to explain it, as in Hofstadter's words, 'in a language we ourselves can fathom'. Let us attempt to explain it from first principles, undisturbed by the fact that we do not possess sufficient knowledge. A priori, an understanding of the organismic function of the brain includes two directions, outside in and inside out. Outside-in is from behavioral function to its neural correlates. The outside includes that what we can observe organisms performing, includes how the world makes it into ourselves, the constraints of physics, and the regularities of interaction between world and organism. The inside-out begins in neuroanatomy and neuronal dynamics and goes through how the brain and the body are rigged for the interaction. To be satisfactory the two directions must meet, regardless of the lack of a well-defined middle.

To be sure, we do not currently possess sufficient knowledge (dissatisfaction is a human characteristic). However, whatever the case with regard to completeness, we can boast some knowledge that would definitely have impressed our noble ancestry. Whatever findings the future might uncover, there are some definite boundary conditions that any explanation will have to meet-boundary conditions known now, indeed, some of them with considerable certainty. Those pieces of knowledge were the kind from which Braitenberg's proposals germinated.

Starting at one end, say for example, neuroanatomy (the "in"), we will not get very far in our explanations if we do not exit at the other side, the world (the "out"). Conversely, starting from the world, we must find out about the regularities of the world that will indisputably determine the physical modes of interaction available, as we and the world, essentially, are made of the same matter, and abide to the same laws. The regularities in interaction between ourselves and the world are determined by our construction, which in turn are determined by the stuff of which the world is made. We are the world. And so to know about ourselves, we also need to know the world.

So much for my attempt to subsume Braitenberg's functional philosophy, which in the present incarnation answers as embodied cognition. But rather than constraining his thoughts to a label, I will reinstate his belief that the regularities in interaction between cognitive systems and the world are determined by physical laws. And that we operate by and large by the same physical laws (Negrello 2011; Merleau-Ponty 1963).

Braitenberg was keenly aware of the two complementary directions, and while being formally trained only in one, as a neuroanatomist, had a grasp of mechanics such as to leave an engineer humbled. It was often between the two extremes where he found the tools that would allow him to frame, describe, and quantify behavioral phenomena. He dwelt in a world of cell counts, fiber tracts, efferent and afferent patterns. But he transcended the data, commuting between levels to tie up both ends of explanation.

It is not incidental that Braitenberg was a burgeoning neuroanatomist in the heyday of cybernetics, which left an indelible mark on his thought. Back in the day, cybernetics had not yet acquired the somewhat mixed reputation it may unfairly carry today. In those days, when Reichardt proposed his model of motion detection, when feedback control was emphasized by Wiener, and Von Holst, Mittelstaedt ushered the environmental loop, the world saw the inception 
of the Rosenblatt's perceptron and the McCullogh-Pitts neuron, Hebb argued cell assemblies, David Marr's outlined his theory of vision, Marvin Minsky, McCarthy, Lashley, Von Foerster, and many others were advancing artificial intelligence, brain theory was teeming with mechanistic insight. Looking back, it is surprising that their explanatory power have often been relegated to the domains of robotics, computer vision, and artificial intelligence. As such, Braitenberg's endeavors appear as a hallmark of his times. But from all his peers he was in the forefront, pushing the boundaries of knowledge, while encouraging his peers to think about the actual brain. And indeed, he often jovially defended the brain, in paragraphs not devoid of sarcasm, when his contemporaries seemed to neglect the grey matter from which their abstractions arose.

Braitenberg started from both ends, neuroanatomy and behavioral function, and hazarded to connect them. His articles often begin with a broad view of the theme, swiftly proceeding to systematically indicating peculiarities of the subject, pointing out necessities, invariances, idiosyncrasies and the remarkable. It was only after a clear disposition of the distinguished features of the system that he attempted to tie loose ends with function. Because,

[...] We will not be able, in most cases, to explain the peculiarities of a certain brain structure by invoking the rules and constraints of the mechanism that synthesize brains out of neurons, but will always have to consider explanations in terms of the function it performs. (Braitenberg 1977)

His articles often end abruptly, as if leaving space for the reader to fill in the gaps.

Acknowledgments Support for this work was provided from The Netherlands Organization for Scientific Research (NWO) NWO-ALWTOP, number 101884. Humble bows go to Fahad Sultan, Jacqueline Griego, Marieke Rohde, John Morris, Hermina Nedelescu, Kevin Hunt, and especially Almut Schüz.

Open Access This article is distributed under the terms of the Creative Commons Attribution License which permits any use, distribution, and reproduction in any medium, provided the original author(s) and the source are credited.

\section{References}

Bower JM (2002) The organization of cerebellar cortical circuitry revisited: implications for function. Ann N Y Acad Sci 978:135-155

Braitenberg V (1954) Zur Frage der anatomischen Veränderungen des Gehirns bei Schizophrenic. Münchener Medizinische Wochenschrift 96:365-367

Braitenberg V (1959) Morphology of nerve nets. Nuovo Cimento 13(2):521-531

Braitenberg V (1962) A note on myeloarchitectonics. J Comp Neurol 118:141-156

Braitenberg V (1965) Taxis, kinesis and decussation. Prog Brain Res $17: 1-13$
Braitenberg V (1977) On the texture of brains: an introduction to neuroanatomy for the cybernetically minded. Springer, New York

Braitenberg V (1977) The concept of symmetry in neuroanatomy. N Y Acad Sci Ann 299:186-196

Braitenberg V (1978) Cortical architectonics: general and areal. In: Brazier MAB, Petsche $\mathrm{H}$ (eds) Architectonics of the cerebral cortex. Raven Press, New York, pp 443-465

Braitenberg V (1983) The cerebellum revisited. J Theoret Neurobiol 2:237-241

Braitenberg V (1984) Vehicles: experiments in synthetic psychology. Bradford Books, Cambridge

Braitenberg V (1987) The cerebellum and the physics of movement: some speculations. Cerebellum and neuronal plasticity. Plenum Press, New York, pp 193-208

Braitenberg V (1991) Information from structure: a sketch of neuroanatomy. Phys Neural Netw 1:107-120

Braitenberg V (1993) Intricacies of movement control: an essay. In: Aertsen A (ed) Brain theory. Elsevier, Oxford, pp 119-127

Braitenberg V (1999) Structural symmetries of brains. Encyclopedia of neuroscience, 2nd edn. Elsevier, Oxford, pp 1-5

Braitenberg V (2001) Brain size and number of neurons: an exercise in synthetic neuroanatomy. J Comput Neurosci 10(1):71-77

Braitenberg V (2002) In defense of the cerebellum. Ann N Y Acad Sci 978:175-183

Braitenberg V, Atwood R (1958) Morphological observations on the cerebellar cortex. J Comp Neurol 109(1):1

Braitenberg V, Braitenberg C (1979) Geometry of orientation columns in the visual cortex. Biol Cybern 33(3):179-186

Braitenberg V, Guglielmotti V, Sada E (1967) Correlation of crystal growth with the staining of axons by the Golgi procedure. Biotech Histochem 42(6):277-283

Braitenberg V, Heck D, Sultan F (1997) The detection and generation of sequences as a key to cerebellar function: experiments and theory. Behav Brain Sci 20(2):229-245; discussion 245-277

Braitenberg V, Kemali M (1970) Exceptions to bilateral symmetry in the epithalamus of lower vertebrates. J Comp Neurol 138(2):137-146

Braitenberg V., Schüz A (1983) Some anatomical comments on the Hippocampus. In: Seifert W (ed) Neurobiology of the hippocampus. Academic Press, London

Braitenberg V, Schüz A (1998) Cortex: statistics and geometry of neuronal connectivity. Springer, New York

Franzius M, Sprekeler H, Wiskott L (2007) Slowness and sparesenss lead to place, head-directino, and spatial-view cells. PLoS Comput Biol 3(8):e166

Jeffery KJ, Burgess N (2006) A metric for the cognitive map: found at last? Trends Cogn Sci 10(1):1-3

Merleau-Ponty M (1963) The structure of behavior. Duquesne University Press, Philadelphia

Negrello M (2011) Invariants of behavior. Constancy and variability in neural systems. Springer, New York

O'Keefe J, Dostrovsky J (1971) The hippocampus as spatial map: preliminary evidence from unit activity in the freely moving rat. Brain Res 34:171-175

Strausfeld N (2009) Brain organization and the origin of insects: an assessment. Proc R Soc B 276(1664): 1929

Strausfeld N, Hansen L, Li Y, Gomez R, Ito K (1998) Evolution, discovery, and interpretations of arthropod mushroom bodies. Learn Mem 5(1):11-37

Sultan F, Braitenberg V (1993) Shapes and sizes of different mammalian cerebella. A study in quantitative comparative neuroanatomy. J Hirnforsch 34(1):79-92

Wyss R, König P, Verschure PFMJ (2003) Invariant representations of visual patterns in a temporal population code. Proc Natl Acad Sci USA 108(1):324-329

Yamazaki T, Tanaka S (2007) The cerebellum as a liquid state machine. Neural Netw 20(3):290-297 